\section{RNEE}

Revista Nicolaita de Estudios Económicos

Nueva Época

Instituto de Investigaciones Económicas y Empresariales
Universidad Michoacana de San Nicolás de Hidalgo

Volumen XV, Número 2, Julio - Diciembre 2020, pp. 119-132

ISSN (Print): 1870 - 5464 ISSN (On-line): 2007 - 9877

DOI: $10.331101 /$ rnee.v15i2.318

(Recibido: 17/septiembre/2020; Aceptado: 27/noviembre/2020)

\title{
El efecto del capital TIC y humano sobre el crecimiento económico
}

\section{The effect of ICT and high skill labor in the economic growth}

\author{
Rodrigo Aliphat a * \\ Oscar Arturo Garcíab
}

\section{Resumen}

Siguiendo la teoría del crecimiento endógeno, este documento estudia, empleando series de tiempo, el efecto de las tasas de crecimiento de capital TIC y empleo calificado sobre el valor agregado para la economía mexicana entre 1990-2019. Los resultados demuestran una relación positiva y significativa entre la incorporación de capital de alta tecnología y trabajadores con mayor nivel educativo en los procesos productivos. Se concluye que, el grado de cualificación del trabajo aumenta la eficiencia en el uso de nuevas tecnologías y tiene un mayor efecto en el crecimiento del PIB; además, se encuentra que la combinación de empleo calificado y TIC crea sinergias que posibilitan la innovación.

Palabras Clave: macroeconomía, crecimiento endógeno, capital humano, TIC, México.

Clasificación JEL: E24, L52, O11, O33

\begin{abstract}
The purpose of this paper is, according to endogenous growth theory, to analyze the effect of the growth rates of ICT capital and skilled employment on the economic growth for the Mexican economy between 1990-2019. Using a time series model, the main result shows a significant and positive relationship between the incorporation of high-tech capital and high skilled labor in the production process. In Mexico, the degree of education level on workers has a major effect on GDP growth, due to the increase in the efficiency in the use of new technologies. Furthermore, we conclude that the combination of skilled employment and ICT creates synergies that enable innovation.
\end{abstract}

Keywords: macroeconomics, endogenous growth, human capital, ICT, Mexico

JEL Codes: E24, L52, O11, O33

$\overline{\text { a }}$ Facultad de Economía, UNAM, E-mail: raliphat@hotmail.com

b Facultad de Economía, UNAM, E-mail: grc.o@outlook.com

* Autor de correspondencia 


\section{Introducción}

A partir de la década de los 90, el crecimiento de las tecnologías de la información y comunicaciones (TIC) de la mano del trabajo calificado se convirtieron en una condición necesaria para la innovación y por lo tanto del crecimiento económico. En México, se observó a nivel sectorial una alta correlación entre la incorporación de TIC y personal calificado con el crecimiento del PIB; sin embargo, entre 1990 y 2018 en promedio el crecimiento del capital TIC fue del $3.8 \%$ respecto del formación bruta de capital fijo (FBKF) total y el personal calificado en las industrias se mantuvo en $13.5 \%$.

A partir de la teoría del crecimiento endógeno, se evalúa el efecto de las tasas de crecimiento de capital TIC y empleo calificado sobre el valor agregado para la economía mexicana en el periodo de 1990-2019. Se busca aportar una función de crecimiento económico en la que se resalte el papel de las TIC y del personal calificado.

El presente documento se divide en cinco secciones, posterior a la introducción en la segunda sección analiza desde la teoría del crecimiento económico endógeno la importancia de las TIC y del CH. La tercera muestra la relación entre las TIC y del capital humano $(\mathrm{CH})$ en el crecimiento económico, y se hace un análisis a nivel sectorial de las variables mencionadas en la estructura productiva mexicana. En la cuarta sección se realiza una estimación econométrica para comprobar en la economía mexicana que las TIC y el CH tienen un efecto positivo en el crecimiento económico. Finalmente, en la quinta sección se presentan las conclusiones.

Por último, los resultados demuestran una relación positiva significativa entre la incorporación de capital de alta tecnología y trabajadores con mayor nivel educativo en los procesos productivos. En México, el grado de calificación del trabajo tiene mayor efecto en el crecimiento del PIB al aumentar la eficiencia en el uso de nuevas tecnologías. Además, la combinación de empleo calificado y TIC crea sinergias que posibilitan la innovación.

\section{Revisión de la literatura teórica}

En la tradición de la teoría del crecimiento endógeno (Romer, 1986, 1990; Lucas, 1988; Grossman y Helpman, 1991; Aghion y Howitt, 1990) se pone de manifiesto que la acumulación de capital humano y físico es esencial en la determinación de la tasa de crecimiento de largo plazo de los países. Estas variables tienen distintos mecanismos de interacción que conducen a explicar la tasa de crecimiento del producto y podrían responder al planteamiento de Ros (2013) acerca de "ipor qué algunas naciones son más pobres que otras y por qué las economías de algunos países crecen más rápido que otras?". En este sentido, el debate teórico que se presenta establece el papel de la formación bruta de capital TIC y el capital humano en el crecimiento de una economía.

La nueva teoría del crecimiento nace como respuesta al desarrollo del modelo neoclásico de crecimiento; donde Solow (1956) concluye rendimientos decrecientes en la función de producción (ecuación 1) y calcula al cambio técnico $\left(A_{t}\right)$ como un residuo de los factores de la producción $(K, L)$. De acuerdo con esta teoría, el crecimiento económico (Y) depende de la velocidad y tamaño del cambio técnico; sin embargo, el cambio técnico $\left(A_{t}\right)$ se considera exógeno por lo que una economía no puede modificarlo.

$$
Y=A_{t} K^{\alpha} L^{\alpha-1}
$$


Solow (1956) concluye que, bajo el planteamiento teórico de la ecuación 1, los países tenderán a un mismo nivel de crecimiento económico. Con el paso del tiempo, economías como México y Estados Unidos, que incluso tienen un tratado de libre comercio, no convergieron en el mismo nivel de crecimiento (Blecker y Esquivel, 2010), por lo que se ha replanteado esta teoría considerando al cambio técnico como endógeno.

Los desarrollos formulados por Romer (1986) y por Lucas (1988), explican que el progreso técnico es endógeno a la economía que se estudia. La contribución conceptual de Romer (1986), señala que la definición de capital debe incluir tanto a la inversión en conocimiento, como a la de bienes de capital físico. Por otra parte, Lucas (1988) establece que es la producción de capital humano y no la de capital físico la que genera crecimiento económico. Snowdon y Vane (2005) señalan que la clave del crecimiento en el modelo endógeno está en la existencia de retornos constantes a escala a la acumulación de capital; además, hacen énfasis en la importancia de la acumulación de tecnología y formación de personal calificado como los factores del crecimiento económico. En el caso de Lucas (1988), el planteamiento comienza estableciendo la existencia de retornos constantes a la tecnología; a diferencia de Solow (1956), establece que el cambio técnico (A) depende del acervo de capital por trabajador (ecuación 2), es decir, se vuelve endógeno.

$$
Y=A K^{\alpha} L^{1-\alpha} ; \quad A=(\widetilde{K} / L)^{\mu}
$$

El planteamiento de Lucas (1988) señala que en la acumulación de capital está implícita la acumulación de conocimiento; esta proposición da lugar a respuestas más robustas por parte de los modelos de crecimiento endógeno de segunda generación. Romer (1990) considera un sector de I+D que genera nuevas variedades de bienes intermedios, los cuales se usan en la función de producción de los bienes finales de consumo, haciendo a la inversión rentable en el tiempo. En ese sentido, a pesar de existir rendimientos decrecientes para la acumulación de la misma variedad de bienes intermedios, la economía puede evitarlos creando nuevas variedades de productos (innovación).

Los modelos de crecimiento endógeno de segunda generación (Aghion y Howitt, 1990; Romer, 1990; Grossman y Helpman, 1991), ponen de manifiesto que el crecimiento en una economía es consecuencia de la innovación. Condicionan el crecimiento de una economía al grado de innovación de sus actividades productivas, ya sea horizontal mediante el desarrollo de variedad de productos (Romer, 1990; y Grossman y Helpman,1991) o vertical a partir de mejoras en la calidad del producto destruyendo la innovación anterior (Aghion y Howitt, 1990).

Para Romer (1990) se crean sinergias entre la acumulación de capital en sus distintas formas y los nuevos desarrollos. Al señalar a las actividades de I+D como causa del crecimiento, argumenta que en el trasfondo existe acumulación de capital humano y físico a partir de la creación de producción intermedia. Romer establece que el crecimiento económico $(Y)$, como se muestra en la ecuación 3, es una función del trabajo físico $(L)$, capital humano $\left(H_{Y}\right)$, y capital físico $\left(x=\left\{x_{i}\right\}_{i=1}^{\infty}\right)$. Este último se interpreta como una variedad de productos intermedios que se crean en un horizonte de tiempo, aunque sólo un número finito de insumos potenciales están disponibles para utilizarse en cualquier momento.

$$
Y\left(H_{Y}, L, x\right)=H_{\mathfrak{x}} L \beta \sum_{\dot{b}+1} x_{\mathfrak{d}_{-}} \alpha_{-} \beta
$$


Para Grossman y Helpman (1991), el crecimiento económico $\left(Y_{t}\right)$ es una función de la variedad de productos intermedios $\left(M_{t}\right)$, sujeto al nivel de capital humano; el trabajo $(L)$ y la producción de bienes finales utilizados en la producción de bienes intermedios $\left(X_{t}\right)$ (ecuación 4).

$$
Y_{t}=M_{t}^{1-\alpha} L^{1-\alpha} X_{t}^{\alpha}
$$

Aghion y Howitt (1990) suponen la existencia de un sector dentro de la economía responsable de generar innovación. Por lo que el crecimiento económico $\left(Y_{t}\right)$ está dado por la productividad del insumo intermedio utilizado en $t\left(A_{t}\right)$ que multiplica al factor trabajo; el insumo intermedio $\left(x_{t}\right)$ es producido por un monopolista utilizando el bien final como insumo. Finalmente, en $t$ existe un emprendedor que realiza un intento por innovar, si tiene éxito, creará una nueva versión del insumo intermedio - con mayor productividad que el desarrollado en " $t-1$ " esto implica que $A_{t}=\gamma A_{t-1} ; \gamma>1$. Si no tiene éxito, entonces la productividad en $t$ será igual a la del periodo anterior $A_{t}=A_{t-1}$.

$$
Y_{t}=\left(A_{t} L\right)^{1-\alpha} x_{t}^{\alpha}
$$

La teoría del crecimiento endógeno señala que se requiere de personal inserto en actividades de I+D y trabajo calificado, es decir, considera al capital humano como el responsable de generar innovaciones. Sin embargo, no diferencia al capital de trabajo del capital que permite innovar (TIC); por lo es importante diferenciar al capital TIC del resto del capital físico.

El auge de las TIC despertó un debate sobre la definición y las clases industriales que se insertan en ella. Como antecedentes, la Organización para la Cooperación y Desarrollo Económicos (OCDE) publicó una lista de las actividades industriales que comprenden a la economía de la información en sus vertientes, tanto del sector TIC, como del sector de contenidos y multimedia (OCDE, 2007). Por otra parte, en la Clasificación Industrial Internacional Uniforme (CIIU), el sector TIC comprende la producción de bienes y servicios de una industria que cumplen con la función de procesamiento de información y comunicación a través de medios electrónicos, incluyendo la transmisión y visualización. Por lo tanto, se define a las TIC como la combinación de las industrias de manufactura y servicios cuyos productos capturan, transmiten o muestran datos e información de manera electrónica; la relevancia de las actividades TIC radica en su característica de facilitar la innovación y en que aparecen tanto en el sector manufacturero, como en el sector servicios.

Pradhan, Malik y Bagchi (2018) sostienen la hipótesis de que la infraestructura TIC es una precondición necesaria para generar crecimiento. El mecanismo de generación de crecimiento económico está en que las TIC son capaces de aprovechar otros tipos de infraestructura y factores de la producción. En específico, el trabajo de Archibugi y Coco (2004) establece una complementariedad entre producción, infraestructura y desarrollo de capital humano, los autores sostienen que sirve de poco el desarrollo en infraestructura TIC u otros si no existe un desarrollo en las habilidades de la población, es decir, del capital humano.

Combinando la importancia del capital humano $(\mathrm{CH})$, expresado por la teoría del crecimiento endógeno, y la importancia del capital TIC, se concluye una extensión de la función de crecimiento económico endógeno $(Y)$, en la que el capital TIC $\left(K_{T I C}\right)$ representa la parte de la formación de capital en constante innovación (ecuación 6).

$$
Y: f\left(C H, K_{T I C}\right)
$$




\section{Situación del capital humano y TIC en México}

A lo largo del siglo XXI, las TIC se han posicionado como un elemento central dentro de los procesos de producción de bienes y servicios en México y el Mundo (Aral, Brynjolfsson y Van Alstyne, 2012; Torrent-Sellens y Ficapal-Cusí, 2010; Gomes, Alves y Silva, 2018). En este nuevo paradigma, los procesos tecnológicos se requieren de trabajo mejor calificado respecto de la industria manufacturera tradicional. El auge en el uso de las TIC desplazó la demanda de empleo en actividades rutinarias e incrementó la necesidad de incorporar trabajadores mejor calificados (Marcolin, Miroudot, y Squicciarini, 2016). La combinación de un mayor uso de TIC y personal calificado llevó a mayores niveles de productividad, incrementó el nivel de competencia entre las industrias y creó un nuevo paradigma en el entendimiento del crecimiento económico.

Los trabajos de Lall, (1992), Pavitt y Bell (1993), Archibugi y Coco (2004), Fagerberg y Srholec (2007) y Fagerberg et al., (2009) demostraron la importancia de la infraestructura TIC en el crecimiento económico; sus análisis bajo el esquema de Capacidades Tecnológicas Nacionales inserta a la aplicación y generación de TIC como determinante de la productividad de las economías. Los beneficios de las TIC estriban en su capacidad para mejorar la conectividad de un país y promover el desarrollo de capital humano.

En el caso de las economías emergentes, incorporar el uso de TIC mejora la competitividad de sus industrias en los mercados mundiales, incrementa sus capacidades productivas y genera incentivo para el desarrollo de capital humano (Pavitt y Bell, 1993). Cuando economías como la mexicana promueven su uso, se crean escenarios propicios para la innovación (Kleis, et. al. 2012), las cuales de acuerdo con Grossman y Helpman, 1991; Aghion y Howitt, 2009 son la base del crecimiento económico contemporáneo.

En la economía mexicana se observa que la formación de capital humano $(\mathrm{CH})$ tiene un efecto positivo en el producto interno bruto (PIB). La correlación positiva entre CH y PIB sugiere incorporar al capital TIC dentro de los procesos productivos bienes y servicios. La combinación de capital TIC y CH genera sinergias en el crecimiento económico. En la gráfica 1a se observa una relación lineal positiva de 0.6750 entre las tasas de crecimiento de personal con alta calificación (CH) y PIB, destaca que las tasas más bajas de crecimiento económico se relacionan con los años en que se contrajo la contratación de personal calificado; de igual forma, la gráfica $1 \mathrm{~b}$ demuestra una relación directa de 0.8273 entre la formación bruta de capital TIC y el crecimiento económico. La evidencia sugiere una relación lineal positiva entre el capital humano y TIC respecto al crecimiento del PIB para la economía mexicana 
Gráfica 1 Correlación entre las tasas de crecimiento del capital humano y TIC respecto del PIB en México. 1990-2017 (Porcentajes)

1a. Relación capital humano - PIB

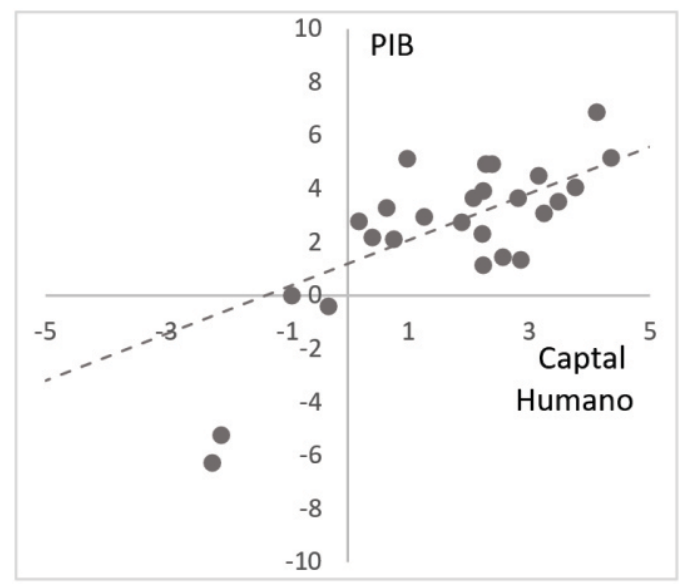

1b. Relación FBKF TIC - PIB

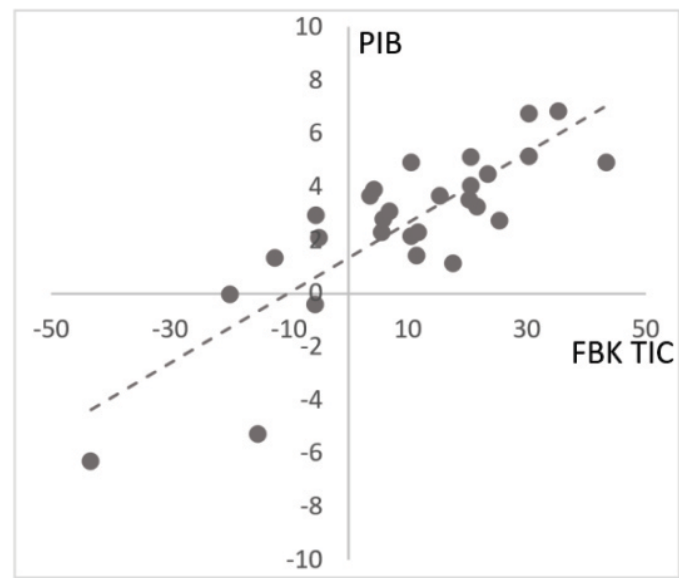

Fuente: Elaboración propia con datos de INEGI (2020)

El crecimiento promedio anual del PIB para el periodo de 1991-2018 fue de $2.6 \%$ mientras que el del capital humano rondó el $1.6 \%$ y la formación de capital TIC de $9.5 \%$; es decir, bajo la lógica de la teoría del crecimiento, en México, el atraso económico respecto a países desarrollados se puede relacionar con el rezago en la incorporación de personal calificado en los procesos productivos.

De acuerdo con los datos de KLEMS (INEGI, 2020), al interior de la esfera productiva la participación de los puestos de trabajo con escolaridad alta representó en 1990 el $13.9 \%$ mientras que para 2018 el porcentaje fue de $13.5 \%$, esto indica que no ha aumentado la proporción de capital humano. Se destaca que en el sector primario el nivel de empleo calificado no alcanza ni el $1 \%$ del total; en contraste, el sector servicios concentra en sus actividades de salud, educación y servicios financieros la mayor cantidad de personal calificado (39.3, 36.1 y $28.5 \%$, respectivamente). Por su parte, el sector de la minería tuvo un mayor crecimiento de su personal calificado al pasar del 5.3 al 8.1\%. Finalmente, el sector manufacturero redujo su personal calificado en $10 \%$ durante el periodo de 1990 a 2018, lo cual es reflejo de la especialización del sector en actividades intensivas en mano de obra poco calificada.

$\mathrm{Al}$ analizar a los sectores productivos por su nivel de inversión en bienes tecnológicos (TIC), se encuentra que el sector de servicios financieros y de seguros, y de información en medios masivos incrementaron sus activos TIC a una tasa promedio entre 1990 y 2018 del 27 y $25 \%$, respectivamente; ambos sectores lideran la digitalización de los sectores productivos mexicanos. En contraparte el sector agrícola primario incrementó su capital TIC a una tasa de $0.3 \%$ anual para el mismo periodo; lo cual explica el cada vez mayor rezago tecnológico del sector primario respecto del sector servicios. Por otra parte, el sector manufacturero aumentó su capital TIC a una tasa del $4 \%$, este sector sigue siendo intensivo en mano de obra (véase cuadro 1). 
Cuadro 1
Personal calificado y capital TIC por sector de actividad económica. 1990-2018 (Porcentajes)

\begin{tabular}{|c|c|c|c|c|c|c|c|c|c|c|}
\hline \multirow[t]{2}{*}{ Tipo de actividad económica } & \multicolumn{5}{|c|}{$\begin{array}{l}\text { Puestos de trabajo ocupados } \\
\text { con escolaridad alta }\end{array}$} & \multicolumn{5}{|c|}{ FBK TIC respecto a la FBK total } \\
\hline & 1990 & 2000 & 2010 & 2018 & Var & 1990 & 2000 & 2010 & 2018 & Prom \\
\hline Total & 13.9 & 14.5 & 13.8 & 13.5 & 0.0 & 1.3 & 3.7 & 4.8 & 5.3 & 3.4 \\
\hline Sector primario (subsector $11^{*}$ ) & 0.8 & 0.7 & 0.8 & 0.7 & 0.0 & 0.0 & 0.0 & 1.1 & 0.7 & 0.3 \\
\hline Sector secundario & 11.9 & 12.0 & 12.8 & 11.8 & -0.1 & 1.7 & 4.5 & 2.0 & 7.1 & 3.3 \\
\hline 21 - Minería & 5.3 & 5.6 & 6.9 & 8.1 & 2.7 & 0.4 & 2.6 & 0.9 & 3.2 & 1.7 \\
\hline $\begin{array}{l}22 \text { - Generación, transmisión y } \\
\text { distribución de energía eléctrica, agua } \\
\text { y de gas al consumidor final }\end{array}$ & 19.6 & 21.6 & 19.2 & 18.7 & -0.9 & 0.4 & 0.4 & 0.5 & 2.5 & 0.7 \\
\hline 23 - Construcción & 14.9 & 14.4 & 15.5 & 15.0 & 0.1 & 1.4 & 3.7 & 1.5 & 19.2 & 5.3 \\
\hline 31-33 - Industrias manufactureras & 10.8 & 10.5 & 10.8 & 9.7 & -1.1 & 2.7 & 5.8 & 2.8 & 7.4 & 4.3 \\
\hline Sector terciario & 17.3 & 17.8 & 16.0 & 16.0 & -1.3 & 1.3 & 3.6 & 6.2 & 4.6 & 3.6 \\
\hline 43-46 - Comercio & 8.1 & 8.9 & 8.9 & 8.9 & 0.8 & 4.0 & 5.4 & 3.7 & 6.8 & 6.3 \\
\hline $\begin{array}{l}\text { 48-49 - Transportes, correos y } \\
\text { almacenamiento }\end{array}$ & 16.6 & 18.7 & 18.5 & 18.3 & 1.6 & 0.3 & 0.7 & 1.2 & 0.9 & 1.2 \\
\hline 51 - Información en medios masivos & 16.2 & 17.0 & 16.5 & 16.7 & 0.5 & 25.7 & 48.9 & 8.6 & 9.0 & 25.3 \\
\hline 52 - Servicios financieros y de seguros & 26.1 & 26.4 & 26.5 & 28.5 & 2.4 & 6.5 & 20.0 & 61.8 & 53.1 & 27.7 \\
\hline $\begin{array}{l}53-55 \text { - Servicios inmobiliarios y de } \\
\text { alquiler de bienes muebles e intangibles, } \\
\text { corporativos }\end{array}$ & 17.9 & 18.2 & 18.1 & 18.6 & 0.6 & 0.1 & 0.3 & 6.5 & 0.1 & 1.0 \\
\hline $\begin{array}{l}54 \text { - Servicios profesionales, científicos y } \\
\text { técnicos }\end{array}$ & 13.8 & 13.7 & 13.8 & 13.9 & 0.0 & 1.4 & -5.6 & 28.1 & 6.6 & 9.9 \\
\hline $\begin{array}{l}56 \text { - Servicios de apoyo a los negocios y } \\
\text { manejo de residuos y desechos, y } \\
\text { servicios de remediación }\end{array}$ & 17.6 & 17.7 & 18.2 & 18.4 & 0.8 & 5.6 & 13.1 & 2.6 & 2.1 & 8.8 \\
\hline 61 - Servicios educativos & 34.5 & 34.5 & 34.5 & 36.1 & 1.6 & -0.3 & 1.0 & 31.2 & 0.9 & 6.2 \\
\hline $\begin{array}{l}62 \text { - Servicios de salud y de asistencia } \\
\text { social }\end{array}$ & 36.8 & 38.7 & 38.7 & 39.3 & 2.5 & 0.2 & 0.5 & 0.7 & 0.4 & 0.5 \\
\hline $\begin{array}{l}71 \text { - Servicios de esparcimiento } \\
\text { culturales y deportivos, y otros servicios } \\
\text { recreativos }\end{array}$ & 15.9 & 16.8 & 16.8 & 17.2 & 1.3 & 1.1 & 2.5 & 1.5 & 7.9 & 2.6 \\
\hline $\begin{array}{l}72 \text { - Alojamiento temporal y de } \\
\text { preparación de alimentos y bebidas }\end{array}$ & 7.0 & 7.4 & 7.4 & 7.2 & 0.2 & 0.6 & 2.2 & 0.2 & 0.7 & 1.0 \\
\hline $\begin{array}{l}81 \text { - Otros servicios excepto actividades } \\
\text { gubernamentales }\end{array}$ & 5.2 & 5.0 & 4.9 & 4.7 & -0.4 & 2.4 & 7.1 & 25.7 & 37.6 & 18.3 \\
\hline $\begin{array}{l}93 \text { - Actividades de Estado, organismos } \\
\text { internacionales y extraterritoriales }\end{array}$ & 29.8 & 31.7 & 17.8 & 17.2 & -12.6 & 0.8 & 1.6 & 3.4 & 3.8 & 2.4 \\
\hline
\end{tabular}

Fuente: Elaboración propia con datos del proyecto KLEMS (INEGI, 2020). 
Al combinar la relación entre inclusión de personal con escolaridad alta y Formación Bruta de Capital TIC (FBKF TIC) se encuentran los sectores productivos mexicanos líderes en la transformación tecnológica del país, como los son el sector financiero y de servicios educativos. Los sectores productivos que requieren con mayor urgencia una política industrial que incentive su transformación tecnológica son el sector primario, servicios de alojamiento y de preparación de bebidas y alimentos, y el de la minería, por sus bajos niveles de personal calificado y de incorporación de capital TIC.

\section{Estimación econométrica de la función: Y=f(CH, KTIC)}

De acuerdo con los hechos estilizados hay una relación estadística entre el crecimiento del PIB respecto la contratación de personal calificado $(\mathrm{CH})$ y la adquisición de capital TIC $\left(K_{T I C}\right)$; esta relación se encuentra fundamentada en la teoría de crecimiento endógeno presentada en la sección II. Por lo que tomando la ecuación (6) se establece la ecuación 7 :

$$
\Delta y=\beta_{0}+\beta_{1} \Delta C H+\beta_{2} \Delta K_{T I C}+\varepsilon
$$

Donde $\Delta y$ representa la tasa de crecimiento del PIB, $\triangle \mathrm{CH}$ representa la tasa de crecimiento del personal ocupado total considerándose como proxy del capital humano y $\Delta K_{T I C}$ representa la FBKF TIC entendida como proxy de bienes tecnológicos.

\section{Construcción de la base de datos}

Los datos utilizados para estimar la relación expresada en la ecuación 7 se obtienen de la base de datos KLEMS (INEGI 2020) y son series anuales de las tasas de crecimiento del PIB, puestos de trabajo ocupados con escolaridad alta y formación bruta de capital fijo por tipo de activo TIC de la economía mexicana para el periodo 1990-2019.

El cuadro 2 muestra la información estadística de $\mathrm{Y}, \mathrm{CH}$ y $\mathrm{K}_{T I C}$, se encuentra que la variable $Y$ tiene una tasa de crecimiento promedio del periodo de $2.59 \%$ mientras que la variable proxy del capital humano tiene una tasa de crecimiento menor equivalente $1.60 \%$ y la variable correspondiente a $K_{\text {TIC }}$ lo hizo a una tasa del $9.52 \%$; al ser el crecimiento de $K_{T I C}$ más de cuatro veces superior a $\mathrm{CH}$, se espera que el crecimiento de $Y$ se encuentra por debajo de su potencial como consecuencia de la falta de incorporación de personal calificado en los procesos producción. 
Cuadro 2

\begin{tabular}{cccccc}
\hline Variable & Promedio & Máximo & Mínimo & $\begin{array}{c}\text { Desv. } \\
\text { Estándar }\end{array}$ & Correlación \\
\hline$Y$ & 0.02594 & 0.06850 & -0.06290 & 0.02940 & 1.00000 \\
$C H$ & 0.01601 & 0.05620 & -0.05080 & 0.02266 & 0.67448 \\
$K_{T I C}$ & 0.09524 & 0.43380 & -0.43330 & 0.18510 & 0.82732 \\
\hline
\end{tabular}

Fuente: Elaboración propia con datos de INEGI (2020).

La correlación observada entre las variables independientes ( $\mathrm{CH}$ y $K_{T I C}$ ) y la dependiente $Y$ es de 0.674 y 0.827 respectivamente. Además, en los años 1995, 2005 y 2009 se encuentra tres outliers que coinciden con la crisis del tequila de 1994, la llamada crisis del “,com” y la financiera de 2008 (Moreno-Brid y Ros, 2018); por lo que la estimación econométrica requerirá una variable dummy para cada año mencionado.

Gráfica 2 Índice de crecimiento de las variables Y, CH y KTIC (año base 1990 = 100)

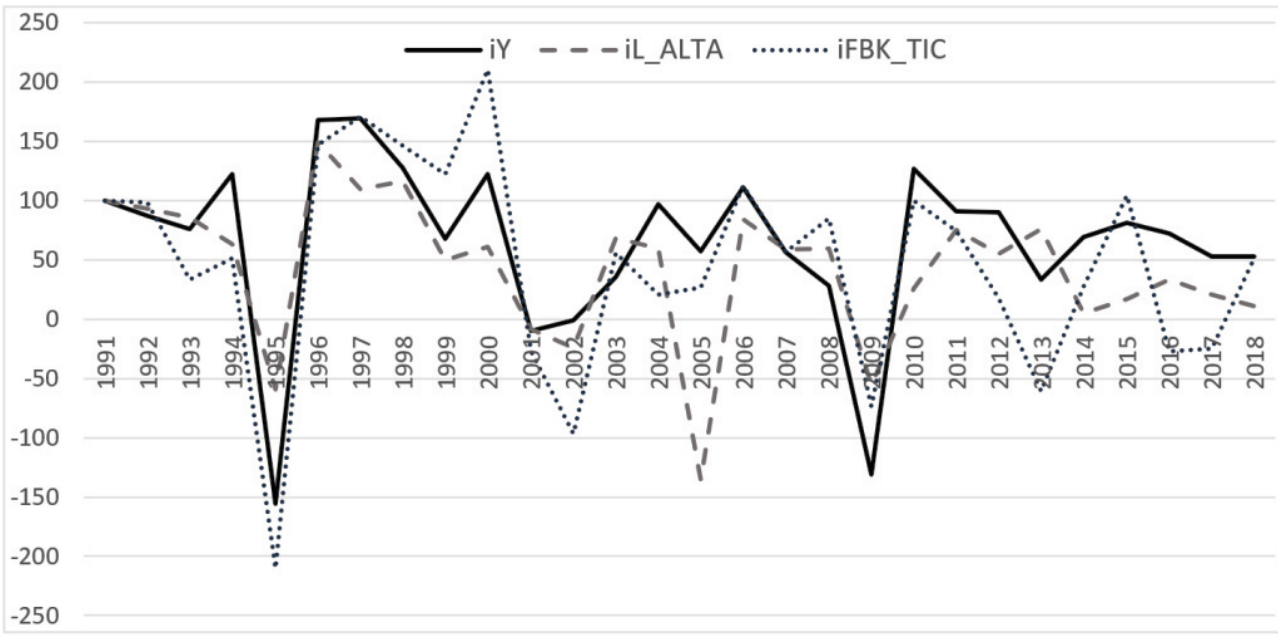

Fuente: Elaboración propia con datos de KLEMS (INEGI, 2020) 
En el cuadro 3, de acuerdo con la prueba de sesgo-curtosis, se observa que las variables tienen una distribución normal. Por lo tanto, una estimación por mínimos cuadrados ordinarios (MCO) de la relación de $\mathrm{CH}$ y $K_{T I C}$ respecto de $Y$ se considera eficiente (Greene, 2017).

Cuadro 3

Prueba de asimetría y curtosis para comprobar normalidad

\begin{tabular}{cccccc}
\hline Variable & Observaciones & Pr(asimetría) & Pr(curtosis) & adj Chi2 (2) & Prob>chi2 \\
\hline$\Delta Y$ & 25 & 0.994 & 0.8744 & 0.03 & 0.9876 \\
$\Delta C H$ & 25 & 0.9845 & 0.8823 & 0.02 & 0.9889 \\
$\Delta K^{\top I C}$ & 25 & 0.6477 & 0.9671 & 0.21 & 0.9001 \\
\hline
\end{tabular}

Fuente: Elaboración propia.

\section{Estimación del modelo}

Siguiendo a Greene (2017), la estimación por MCO considera una relación lineal entre las variables independientes y la dependiente (véase gráfica 1). En el cuadro 4 se presentan los resultados de la estimación. Se encuentra que el modelo tiene un buen ajuste $[\mathrm{R}$-cuadrado $=0.8458]$ lo cual al combinarse con los resultados de la prueba $\mathrm{F}$ [Pr Prueba $\mathrm{F}=0.00000$ ] permiten determinar que el modelo en su conjunto es significativo. De manera individual se encuentra que las variables $\Delta \mathrm{CH}$ y $\Delta K_{T I C}$ tienen signo positivo y son estadísticamente significativas; por lo tanto, se establece que se comportan de acuerdo con el planteamiento teórico hecho en la sección II.

Cuadro 4 Resultados de estimar por $\mathrm{MCO}$ de la relación entre $\Delta \mathrm{CH}$ y $\Delta K_{T I C}$ respecto $\Delta Y$

\begin{tabular}{|c|c|c|c|c|}
\hline \multicolumn{2}{|c|}{$\begin{array}{c}\text { Prueba } F \\
\text { Bondad de Ajuste }\end{array}$} & \multirow{2}{*}{$\begin{array}{c}F(5,22)=30.63 \\
R \text {-squared }=0.8744 \\
\text { Error Estándar }\end{array}$} & \multicolumn{2}{|c|}{$\begin{array}{c}\text { Prob }>F=0.00000 \\
\text { Adj } R \text {-squared }=0.8458\end{array}$} \\
\hline Variable & Coeficiente & & Estadístico $T$ & $P$-value \\
\hline$\Delta C H$ & 0.44415 & 0.18971 & 2.34 & 0.004 \\
\hline$\Delta K_{T I C}$ & 0.06204 & 0.01927 & 3.22 & 0.011 \\
\hline$D 1$ & -0.04126 & 0.0147562 & -2.80 & 0.137 \\
\hline$D 2$ & 0.02703 & 0.0175176 & 1.54 & 0.001 \\
\hline D3 & -0.04938 & 0.0134635 & -3.67 & 0.001 \\
\hline Cons & 0.01519 & 0.00403 & 3.77 & 0.001 \\
\hline
\end{tabular}

Fuente: Elaboración propia. 


\section{Validación del modelo}

Para comprobar la correcta estimación del modelo se realizan las pruebas de normalidad, correlación y homocedasticidad sobre los errores (Greene, 2017). En el cuadro 5 se presentan los resultados de las pruebas aplicadas sobre los residuos; se encuentra que los resultados se distribuyen de manera normal, no hay correlación entre ellos y son homocedásticos; por lo tanto, el modelo esta correctamente estimado.

Cuadro 5

Validación del modelo

\begin{tabular}{lc}
\multicolumn{1}{c}{ Prueba } & Prob $>$ z \\
\hline Pruebas de normalidad [Ho: distribución normal] & \\
Asimetría-curtósis & 0.7856 \\
Saphiro-wilk & 0.5646 \\
Pruebas de independencia [Ho: no correlación] & $(6,28) 1.91$ \\
Prueba Durbin Watson & 0.7967 \\
Breusch-Godfrey & \\
Varianza constante de los errores (HO: Homocedasticidad) & 0.3452 \\
Prueba Breusch-Pagan & 0.7301 \\
Prueba White &
\end{tabular}

Fuente: Elaboración propia.

Por último, la gráfica 3 compara los valores observados $\left(Y_{o b s}\right)$ respecto de los estimados por el modelo para la variable estimada $\left(Y_{e s t}\right)$. Con los patrones establecidos se concluye que el modelo predice de manera eficiente los valores para la variable dependiente $Y$; por lo tanto, se valida la relación teórica expresada en la ecuación 6. 


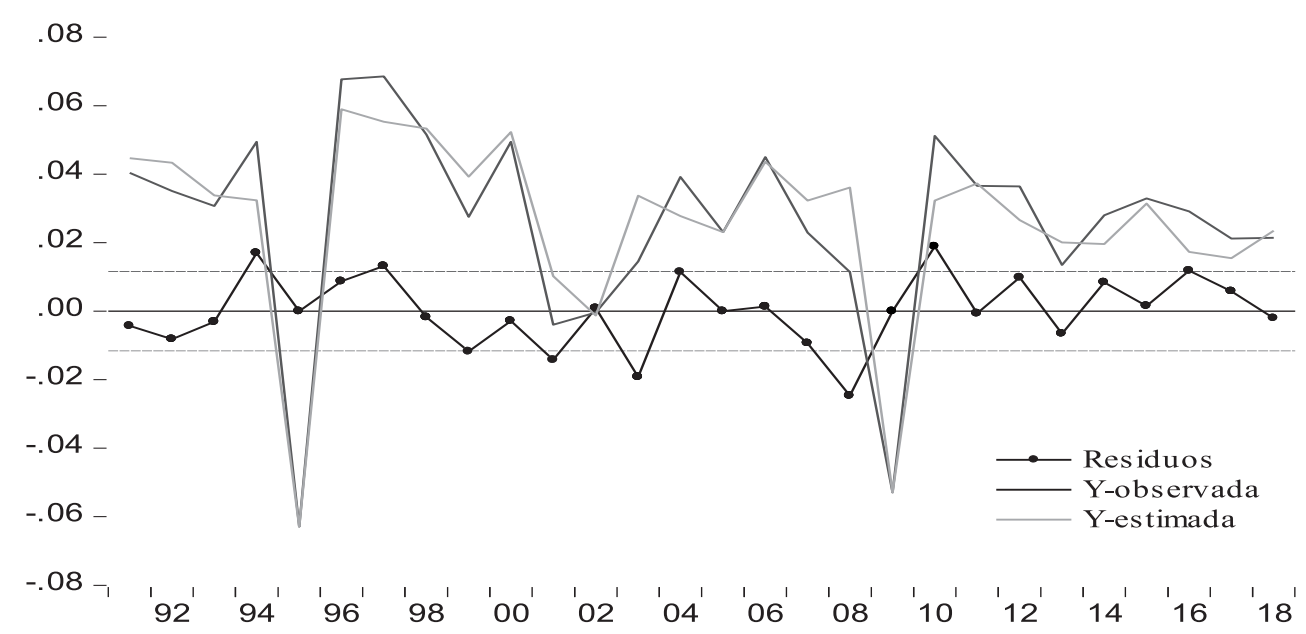

Fuente: Elaboración propia.

Interpretación de resultados

Se encuentra que el efecto de un incremento del $1 \%$ de $\mathrm{CH}$, genera un crecimiento de $0.4441 \%$ sobre el crecimiento del PIB, valor superior al observado para un incremento de la FBKF TIC, que es de 0.0621\%. Estos resultados son lógicos dado que la tasa de crecimiento de $K_{T I C}$ ha sido superior al del personal ocupado calificado (véase cuadro 1). Se concluye que en Mexico hay un crecimiento del PIB por debajo de su potencial debido a la falta de personal ocupado calificado en la estructura productiva nacional.

\section{Conclusiones}

De acuerdo, con los resultados obtenidos de la estimación econométrica, se valida la hipótesis sobre la relación positiva entre el capital humano y TIC respecto del PIB, por lo que este resultado confirma el planteamiento hecho por la teoría del crecimiento endógeno, y además expone la importancia de la formación de capital TIC dentro de los nuevos procesos de producción.

La información estadística obtenida, de los sectores productivos mexicanos y del modelo econométrico, demuestra la necesidad de incrementar la formación y eventual incorporación de capital humano dentro de los procesos de producción. Se observa que el efecto en el PIB de aumentar el nivel de capital TIC es menor cuando no se acompaña de una mayor proporción de personal calificado.

Se encuentra que, dentro de los lineamientos de política industrial como la mexicana, es importante considerar que las economías emergentes le den importancia al binomio capital humano-TIC, ya que además de aumentar su competitividad global, también dinamizarán el crecimiento del cambio técnico y por lo tanto la innovación de los sectores industriales nacionales. 


\section{Referencias}

Aghion, P. y Howitt, P., 1990. A Model of Growth Through Creative Destruction (Working Paper No. 3223), Working Paper Series. National Bureau of Economic Research. DOI: 10.3386/w3223

Aral, S., Brynjolfsson, E. y Van Alstyne, M., 2012. Information, technology, and information worker productivity. Information Systems Research, 23(3-part-2): 849-867. DOI: 10.1287/isre.1110.0408

Archibugi, D. y Coco, A., 2004. A New Indicator of Technological Capabilities for Developed and Developing Countries (ArCo). World Development, 32: 629-654. DOI: 10.1016/j.worlddev.2003.10.008

Arvanitis, S., Loukis, E.N. y Diamantopoulou, V., 2016. Are ICT, workplace organization, and human capital relevant for innovation? A comparative Swiss/Greek study. International Journal of the Economics of Business, 23(3): 319-349. DOI: 10.1080/13571516.2016.1186385

Blecker, R.A. and Esquivel, G., 2010. NAFTA, Trade and Development (Robert A. Blecker and Gerardo Esquivel). In CESifo forum (Vol. 11, No. 4, pp. 17-30). München: ifo Institut für Wirtschaftsforschung an der Universität München

Gomes, A.O., Alves, S.T. y Silva, J.T., 2018. Effects of investment in information and communication technologies on productivity of courts in Brazil. Government Information Quarterly, 35(3): 480-490. DOI: 10.1016/j.giq.2018.06.002

Greene, W. H. 2018. Econometric analysis, Nueva York, Pearson

Grossman, G.M., Helpman, E., 1991. Innovation and Growth in the Global Economy. Boston: MIT Press.

INEGI, 2020. Sistema de Cuentas Nacionales de México. Productividad Total de los Factores Modelo KLEMS. México: INEGI.

Kleis, L., Chwelos, P., Ramirez, R. V., y Cockburn, I., 2012. Information technology and intangible output: The impact of IT investment on innovation productivity. Information Systems Research, 23 (1): 42-59. DOI: $10.1287 /$ isre. 1100.0338

Lucas, R., 1988. On the mechanics of economic development. Journal of Monetary Economics 22: 3-42. DOI: 10.1016/0304-3932(88)90168-7

Marcolin, L., Miroudot, S. y Squicciarini, M., 2016. The Routine Content of Occupations (Trade Policy Papers, No. 188), OECD Publishing. Organization for Economic Cooperation and Development. DOI: 10.1787/5jm0mq86fljg-en

Moreno-Brid, J. C. y Ros, J., 2009. Desarrollo y crecimiento en l a economía mexicana: una perspectiva histórica. México: Fondo de Cultura Económica.

ONU, 2009. Clasificación Industrial Internacional Uniforme de todas las actividades económicas (CIIU) Rev 4. Naciones Unidas, Nueva York.

OCDE, 2007. Information Economy - Sector Definitions Based on the International Standard Industry Classification (ISIC 4) (No. (Núm. DSTI/ICCP/IIS(2006)2/FINAL)). Organización para la Cooperación y Desarrollo Económicos, París.

Pradhan, R.P., Mallik, G. y Bagchi, T.P., 2018. Information Communication Technology (ICT) Infrastructure and Economic Growth: A causality evinced by cross-country panel data. IIMB Management Review 30: 91-103. DOI: 10.1016/j.iimb.2018.01.001

Romer, P., 1986. Increasing Returns and Long-Run Growth. Journal of Political Economy 94: 1002-1037. DOI: $10.1086 / 261420$ 
Romer, P.M., 1990. Endogenous Technological Change. Journal of Political Economy 98, S71-S102. DOI: $10.1086 / 261725$

Ros, J., 2013. Rethinking Economic Development, Growth, and Institutions. Oxford: OUP Oxford.

Snowdon, B. y Vane, H., 2005. Modern Macroeconomics: Its Origins, Development and Current State. Cheltelham: Edward Elgar Publishing.

Solow, R., 1956. A Contribution to the Theory of Economic Growth. The Quarterly Journal of Economics 70 : 65-94. DOI: $10.2307 / 1884513$

Torrent-Sellens, J. y Ficapal-Cusí, P., 2010. ¿Nuevas fuentes co-innovadoras de la productividad empresarial?. INNOVAR. Revista de Ciencias Administrativas y Sociales, 20(38), pp.111-124. Disponible en: redalyc.org/articulo.oa?id=818/81819024009 